\title{
Mode of action of creatinine on colour of cuprous oxide precipitate in Benedict's qualitative sugar test
}

\author{
B. K. SUR, R. K. SHUKLA, AND V. S. AGASHE \\ From the Department of Biochemistry, GSVM Medical College, Kanpur, India
}

SYNOPSIS Creatinine appears to alter the colour and bulky nature of the cuprous oxide precipitat $\vec{e}$ not by altering the chemical composition of the precipitate but by the physical process of retardation $\vec{\omega}$ of the growth of newly formed yellow cuprous oxide crystals to large red crystals by adhering to their surface and blocking some sites for further crystal growth.

It has been shown (Sur, Shukla, and Agashe, 1972) that the colour of the precipitate formed during Benedict's qualitative test with urinary reducing sugar is dependent not only on the concentration of glucose but also on that of creatinine. This could occur due to the chemical alteration of the precipitate or to some physical change in the nature of the particles. Results of experiments designed to elucidate the mode of action of creatinine are presented.

\section{Materials and Methods}

BENEDICT'S TEST

This was carried out as described earlier (Sur et al, 1972). When necessary the test was carried out in centrifuge tubes.

Creatinine in cuprous oxide precipitate

Cuprous oxide reduces picric acid and hence interferes in creatinine estimation by the picric acid test and must be separated first. The washed precipitate was dissolved in $\mathrm{N} / 5 \mathrm{HCl}$ with slight warming. Copper was removed from an aliquot by adding sufficient $3 \% \mathrm{NaOH}$. After centrifugation an aliquot from the supernatant was used for creatinine estimation by the picric acid method as described by Bonsnes (1945).

\section{Copper}

Copper was estimated on an aliquot after suitable dilution by the biscyclohexanoneoxalyl-dihydrazone method as described for ash (Natelson, 1957) but without adding thiocyanate and amyl alcohol. Copper precipitated from Benedict's solution was also estimated by difference by direct colorimetry on the centrifuged supernatant after six-fold dilution Received for publication 17 August 1972. with water using a 66 red filter in a Klett Summersorio photoelectric colorimeter.

\section{Results}

PARTICLE SIZE

If the particles of the precipitate are viewed aftec suitable dilution with water under an ordinary microscope, it is readily seen that the red particles are relatively large and settle down on the slide. The particles appear as squares (each side about $2 \mu$ ) $\rightarrow$ p circles frequently occurring as small clusters. particles of the yellow precipitate appear very small do not readily settle down, continue to execute कै zigzag movement, and often occur as large branched clusters. The orange particles have an intermediate size. The colour of the precipitate appears to be related to particle size.

Other observations are presented in Table I and Figures 1 and 2.

\begin{tabular}{lllll}
\hline \multicolumn{4}{c}{ Glucose $(\%)$} \\
\cline { 2 - 5 } & 0.5 & 1.0 & 1.5 & 2.0 \\
\hline Creatinine added $(\mu \mathrm{g})$ & 400 & 400 & 400 & 400 \\
Creatinine in precipitate $(\mu \mathrm{g})$ & 174 & 276 & 363 & 382 \\
Copper in precipitate $(\mu \mathrm{g})$ & 4800 & 4900 & 13400 & 17500 \\
Creatinine/copper (molar ratio) & $1: 48$ & $1: 58$ & $1: 66$ & $1: 84$ \\
\hline
\end{tabular}

Table Molar ratio of creatinine:copper in cuprous oxide precipitate

\section{Discussion}

REDUCING ACTION

Benedict's solution is said to be susceptible tơ reduction by creatinine (Harrison, 1957; Frankel, 0 1963) although to a lesser extent than in Fehling? 


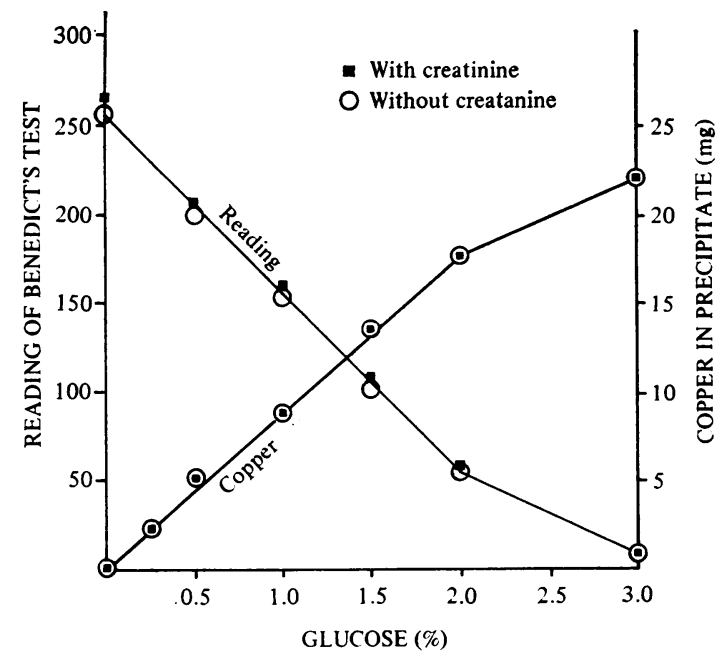

Fig. 1 Graph showing that creatinine (0.2\%) had no effect on the extent of reduction by glucose in Benedict's test. Benedict's solution left after testing was centrifuged and the supernatant diluted six times before colorimetry

solution. Figure 1 shows that creatinine does not have any measurable effect on the extent of reduction of cupric ions at various concentrations of glucose. Hence the influence of creatinine on the colour of the precipitate is not likely to be related to any reducing power of creatinine. Incidentally, this also shows that the optical density of the supernatant of the Benedict's solution is inversely proportional to the amount of glucose in the test sample, and hence this can form the basis of an accurate and simple method of estimating urinary glucose and is totally independent of creatinine concentration. The method can be extended to high-sugar specimens by reducing the usual volume of $0.4 \mathrm{ml}$ of urine.

TIME OF COMMENCEMENT OF PRECIPITATION Any reducing action of creatinine should decrease the time of commencement of precipitation. Figure 2 shows that creatinine actually increases this time, a delaying action of which is more effective at low concentration of glucose $(0.25 \%$ and $0.5 \%)$ than at higher concentrations. Figure 2 further shows that time of commencement of precipitation cannot be made the basis for a reliable method for semiquantitative assay of urine sugar.

\section{CREATININE IN THE PRECIPITATE}

That creatinine appears in the precipitate is shown by the following facts.

1 Micro-Kjeldahl determinations demonstrate the presence of nitrogen in precipitates obtained

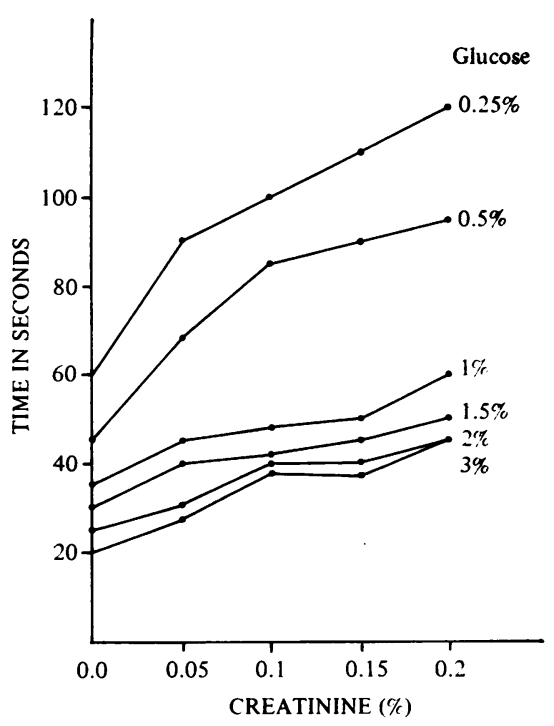

Fig. 2 Influence of creatinine on time of commencement of precipitation of cuprous oxide

with pure solution of glucose, creatinine, and Benedict's solution. Apart from creatinine none of the other constituents used contain nitrogen. This is therefore an unequivocal proof of the presence of nitrogen of creatinine in the precipitate.

2 Creatinine can be estimated in the precipitate after separating copper by the picric acid test (Table).

3 The absorption spectra of the chromogen developed with picric acid was identical with that obtained with pure creatinine solution similarly treated with picric acid.

\section{CUPROUS CREATININE}

Samson (1939) attributed the great sensitivity of Benedict's test at very low concentrations of urinary glucose $(0.05-0.3 \%)$ to the formation of a simple 1:1 additive compound of cuprous creatinine $\mathrm{C}_{4} \mathrm{H}_{7} \mathrm{ON}_{3}$. CuOH. His belief that the insolubility of cuprous creatinine in carbonate solutions explained the sensitivity of Benedict's test is untenable on the following grounds. (1) It can be calculated from the solubility data presented by Samson that the concentration of urinary creatinine would have to exceed $0.7 \%$ before precipitation of cuprous creatinine could commence in boiling Benedict's solution. Such a high concentration of creatinine does not occur in human urine. (2) Cuprous creatinine is highly soluble in water. Yet it cannot be leached out with water from the precipitate obtained in Benedict's test. (3) Samson prepared 
cuprous creatinine under conditions entirely different from those of Benedict's test.

CREATININE : COPPER MOLAR RATIO

The Table shows that the molar ratio of creatinine: copper in the precipitate is variable and very low, pointing to the possibility that creatinine may act through some surface action rather than by forming a chemical compound.

\section{CRYSTAL GROWTH}

If a pure solution of glucose is boiled with Benedict's solution, the precipitate is invariably of a red colour. If the reduction is observed with great care, using a transparent water bath, a transient green colour is seen as the first visible commencement of reduction, presumably due to formation of yellow crystals. As far as we know this observation has never been recorded in the literature. That a yellow precipitate is actually formed before it rapidly changes to the red form can be shown by arresting the growth of the crystals by pouring the boiling solution, immediately on the appearance of the transient green colour, onto a mixture of ice and water. The precipitate is yellow and under the microscope the crystals appear much smaller than red crystals. Thus it appears that in the absence of creatinine, tiny yellow crystals of cuprous oxide are first formed and then they grow very rapidly into large red crystals. Creatinine in some way retards this normal process of rapid growth of cuprous oxide crystals. This could happen if creatinine or cuprous creatinine is adsorbed or fixed on the surface of freshly formed yellow crystals blocking some sites for their ordered growth to red crystals. Our preliminary observation $\overrightarrow{\overrightarrow{\mid}}$ on differential extraction of creatinine and coppero by dilute $\mathrm{HCl}$ of successively increasing concen-흠 tration do show that creatinine is situated closer to the surface of the crystals than in the interior. The greatly increased surface of the precipitate and the extended branched clustering of the crystals would ${ }^{\text {is }}$ make the precipitate more bulky and the test more $\vec{\circ}$ sensitive at low concentrations of glucose.

We thank Dr N. K. Bhide of the All-India Institute of Medical Sciences, New Delhi, for drawing ouri attention to the forgotten work of Samson (1939).

References

Bonsnes, R. W., and Taussky, H. H. (1945). On the colorimetrico determination of creatinine by the Jaffe reaction. J. biol.J

Chem., 158, 581-591.
Frankel, S. (1963). Urine analysis: chemical examination. In $\vec{O}$ Gradwohl's Clinical Laboratory Methods and Diagnosis, 6th ed., vol. 2, edited by S. Frankel and S. Reitman. Mosby, St. Louis.

Harrison, G. A. (1957). Chemical Methods in Clinical Medicine, 4th ed., p. 160. Churchill, London.

Natelson, S. (1957). Microtechniques of Clinical Chemistry for theRoutine Laboratory, p. 183. Thomas, Springfield, Illinois.

Samson, M. (1939). The relation of cuprous creatinine to tests \&nN sugar in urine. J. Amer. chem. Soc., 61, 2389-2392.

Sur, B. K., Shukla, R. K., and Agashe, V. S. (1972). Role of creatin e and histidine in Benedict's qualitative test for reducing sugar in urine. J. clin. Path., 25, 892-895. 